\title{
Influence of Various Media on the Dissolution Profiles of Immediate-Release Quetiapine Tablets in India
}

Zubin Shah* and Vaishali Londhe

Shobhaben Pratapbhai Patel School of Pharmacy and Technology Management, SVKM's NMIMS, Mumbai, India

e-mail: zubin131@gmail.com

\begin{abstract}
Dissolution testing was performed to evaluate the drug release from tablets. To mimic actual in vivo conditions, various foods and beverages were added to the dissolution media, and the release profiles were investigated. Indian people tend to have milk, curds, or eggs during their meals and then take their medications immediately after meals, whereas Americans tend to take medications with beverages. To evaluate drug release profiles in the presence and absence of these foods and beverages, in vitro dissolution testing was conducted using three different Indian quetiapine fumarate tablet brands. The effect on drug release was evaluated by adding $100 \mathrm{~mL}$ milk (3.1\% protein), $100 \mathrm{~g}$ curd (4.1\% protein), and $50 \mathrm{~g}$ raw and boiled egg white individually to $0.1 \mathrm{~N} \mathrm{HCl}$, and the release was compared with that in only $0.1 \mathrm{~N} \mathrm{HCl}$ as per the Indian Pharmacopeia (IP) (1). The study was conducted for $3 \mathrm{~h}$ in triplicate, and the results show a significant difference among the brands in each medium with food. The maximum release obtained for each brand in $0.1 \mathrm{~N} \mathrm{HCl}$ was $90 \%$; in the presence of milk, curd, raw egg, and boiled egg it was approximately $7.06 \%, 2.42 \%, 29.56 \%, 24.08 \%$, respectively, indicating that the drug was unavailable. Studies were also conducted with beverages and antacids; $200 \mathrm{~mL}$ of Thums Up, Sprite, and ENO were added individually to $0.1 \mathrm{~N} \mathrm{HCl}$ and the release results compared. Results indicate a decrease of around $15 \%$ in availability in the presence of beverages and a $37 \%$ decrease in the presence of antacids. The results were statistically compared using one-way ANOVA at its $Q$ value (45 min).
\end{abstract}

KEYWORDS: Quetiapine tablets; in vitro dissolution; milk; curd; egg; beverages; antacids.

\section{INTRODUCTION}

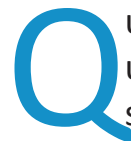

uetiapine is classified as BCS Class 2 and is used to treat atypical antipsychotic conditions, schizophrenia, and bipolar disorders (2). Studies (3) for the effect of beverages on the in vitro disintegration of immediate-release (IR) pain medications show a hindrance in drug absorption; therefore, similar dissolution studies were carried out on quetiapine fumarate IR tablets to check the effect of food and beverages on drug release as the drug is reported to be highly protein bound in vivo.

Most drugs are taken orally, but it is difficult to understand how these drugs are absorbed from the gastrointestinal tract into the body. The effectiveness of dosage forms such as tablets and capsules depends on drug dissolution in the fluids of the gastrointestinal tract prior to absorption into the systemic circulation. The rate of dissolution of the tablet or capsule is therefore crucial.

One of the problems facing the pharmaceutical industry is the optimization of the amount of drug available to the body (i.e., bioavailability). Drug release in the human body can be measured in vivo by measuring the plasma or urine concentration in the subject. However, there are

*Corresponding author.

Dissolution Technologies | FEBRUARY 2016 certain obvious impracticalities involved in employing such techniques on a routine basis. These difficulties have led to the introduction of official in vitro tests that are now rigorously and comprehensively defined in the respective pharmacopoeia.

Tablet dissolution is a standardized way of measuring the rate of drug release from a dosage form. The basic functions of the dissolution test are:

1. Optimize therapeutic effectiveness during product development and stability assessment stages.

2. Routinely monitor production quality to ensure uniformity among production lots.

3. Assess bioequivalence to show that products from distinct batches or from different plants have the same biological activity.

4. Aid in the prediction of bioavailability (4).

The main aim of the study was to check the effect of food proteins and beverages on the drug release profile of quetiapine fumarate tablet, so in vitro dissolution studies 
were performed. The problem with the compendial test media is that they do not accurately mimic the highly variable and dynamic environment of the human gut. Gastric conditions (i.e., condition in which the tablet is taken by the patient) were mimicked by the addition of $100 \mathrm{~g}$ of milk (3.1\% protein), $100 \mathrm{~g}$ curd (4.1\% protein), $50 \mathrm{~g}$ raw egg white, and $50 \mathrm{~g}$ boiled egg white to $0.1 \mathrm{~N}$ hydrochloric acid solution. In addition, the effect of drug release was checked in the presence of Thums Up (strongly carbonated drink), Sprite (mildly carbonated drink), and ENO (antacid effervescent granules). The selection of food was based upon the dietary habits of Indian and American people.

\section{EXPERIMENTAL}

\section{Materials}

Quetiapine fumarate $100-\mathrm{mg}$ tablets of three different brands (A, Batch No. DR 3251; B, Batch No. CV30A004; and C, Batch No. BSN2933) were purchased in Mumbai, India. Quetiapine fumarate IP was obtained as a gift sample from Microlabs, Mumbai, India. Milk (3.1\% protein) and curd $(4.1 \%$ protein) containing $3 \%$ fat were obtained from Amul, Mumbai, India. Eggs were obtained from a poultry house in Mumbai, India. Thums Up and Sprite were purchased from Coca Cola, India. ENO (Regular) was obtained from GlaxoSmithKline Pharmaceuticals Limited, India.

Hydrochloric acid, sodium acetate, sodium chloride, and glacial acetic acid were all analytical reagent grade and were obtained from Research Lab, India.

\section{Instruments}

Apparatus 1 and 2 (Electrolab, model TDT-08L) were used for performing in vitro drug release studies. A pH meter (Lab India, Pico+ model) was used to measure pH. An Eppendorf Remi model RM-12 CBL centrifuge was used for centrifugation of samples. A UV-vis spectrophotometer (Lamda 25, Perkin Elmer) was used for the determination of drug released.

\section{Dissolution Studies}

Dissolution studies were conducted as per IP (1) using Apparatus 2 and $900 \mathrm{~mL}$ of $0.1 \mathrm{~N} \mathrm{HCl}$ as the medium at $37^{\circ} \mathrm{C}$ and $50 \mathrm{rpm}$. Readings were taken at predetermined intervals at $248 \mathrm{~nm}$ using a UV spectrophotometer in which the specific dissolution medium was used as a blank. The release profiles of the brands were compared statistically using one-way ANOVA. The dissolution profiles were calculated using linearity plots in individual media.

\section{Sample Treatment}

In the case of milk, curd, and egg media, sample treatment was required before taking the readings. The samples were placed in tubes and centrifuged at $12000 \mathrm{rpm}$ at 4 ${ }^{\circ} \mathrm{C}$ for $15 \mathrm{~min}$. After treatment, coagulation of milk and curd protein was observed at the top of the tube, and egg protein settled at the bottom. Thereafter, clear medium containing free drug was taken from each tube with the help of a syringe and transferred to the microcuvettes, and readings were taken at $248 \mathrm{~nm}$. No interference was observed at $248 \mathrm{~nm}$ in the presence of food.

\section{Dissolution Procedure Modification}

The studies were modified for clear visibility of tablets in the presence of food. To determine whether or not tablet residue remained at the end of dissolution, the studies were conducted in Apparatus 1 at 100 rpm.

\section{Medium Preparation}

Various foods were added to $0.1 \mathrm{~N} \mathrm{HCl}(\mathrm{pH} 1.2)$ as specified above. Fed-state gastric simulated fluid (FeSSGF) was prepared as per FDA (5), and the pH was adjusted to 5.

Dissolution testing was performed in $0.1 \mathrm{~N} \mathrm{HCl}$ with milk (pH 1.3), $0.1 \mathrm{~N} \mathrm{HCl}$ with curd (pH 1.3), FeSSGF (pH 6.5), $0.1 \mathrm{~N} \mathrm{HCl}$ with raw egg white $(\mathrm{pH} 1.3)$, and $0.1 \mathrm{~N} \mathrm{HCl}$ with boiled egg white $(\mathrm{pH}$ 1.3). The drug release profiles of three different brands of quetiapine fumarate marketed tablets $(100 \mathrm{mg}$ ) were investigated in triplicate using Apparatus 1 and $900 \mathrm{~mL}$ of medium at $37^{\circ} \mathrm{C}$ and $100 \mathrm{rpm}$. Ten-milliliter samples were withdrawn at regular intervals for $3 \mathrm{~h}$, and the volume was kept constant by replacement with an equal volume of fresh buffer/medium at $37^{\circ} \mathrm{C}$. Absorbances were measured at predetermined intervals by UV at $248 \mathrm{~nm}$. The method was modified slightly for milk, curd, and FeSSGF by adding glacial acetic acid to precipitate casein.

Dissolution profiles for various media were calculated, and the available free drug was found by calculating $A U C$ using the trapezoidal rule. One-way ANOVA at $45 \mathrm{~min}$ ( $Q$ value for $0.1 \mathrm{~N} \mathrm{HCl}$ ) (1) was applied to determine if there was a significant difference in the release among brands.

\section{Dissolution in the Presence of Beverages and Antacids} For dissolution in the presence of beverages and antacids, $200 \mathrm{~mL}$ of each beverage (Thums Up, Sprite, and ENO) was added to $700 \mathrm{~mL}$ of $0.1 \mathrm{~N} \mathrm{HCl}$, and dissolution studies were performed as per IP. 


\section{RESULTS AND DISCUSSION}

The release profile of drug quetiapine fumarate was analyzed using a UV spectrophotometer, and the absorbance was measured at $248 \mathrm{~nm}$. The linearity plot gave an equation of $y=0.044 \mathrm{x}-0.0201, R^{2}=1$. The dissolution profiles of the three brands in $0.1 \mathrm{~N} \mathrm{HCl}$ using Apparatus 1 and 2 were the same. The release was $>85 \%$ for all three brands, and all passed the IP criterion (NLT $70 \%$ in $45 \mathrm{~min}$ ). The RSD values indicate that there was not much variation among the tablets of each specific brand. One-way ANOVA was applied, and at the $Q$ point, there was no significant difference $(p=0.352)$ in the release among the brands. The AUC for all three brands was $100 \%$ in $0.1 \mathrm{~N} \mathrm{HCl}$. Furthermore, the drug release profiles drastically decreased when the dissolution studies were carried out with the addition of $100 \mathrm{~mL}$ of milk. The dissolution release was $<8 \%$ for all three brands. There was much variation among the individual tablets of each brand; it can be correlated that the foaming caused by milk interferes in the disintegration of the tablets. The AUC values of Brands A, B, and C are $9.3 \%, 7.9 \%$, and $14 \%$ available free drug, respectively. The release of drug at its $Q$ value was compared using one-way ANOVA, and a significant difference $(p=0.000)$ was found. The dissolution studies were then carried out with the addition of $100 \mathrm{~g}$ curd. The drug release profile decreased more with curd than with milk. The dissolution release was $<2.5 \%$ for all three brands. There was much variation among the individual tablets for each brand; it can be correlated that the foaming caused by milk interferes in the disintegration of the tablets. A comparison of the AUC values shows that the amount of available free drug for Brands $A, B$, and $C$ was $6.5 \%, 4.5 \%$, and $4.1 \%$, respectively. The release of drug at its $Q$ point was compared using one-way ANOVA, and there was a significant difference $(p=0.007)$. The dissolution studies were then performed in FeSSGF, and all the three brands did not disintegrate even after $1 \mathrm{~h}$ (Figure 1).

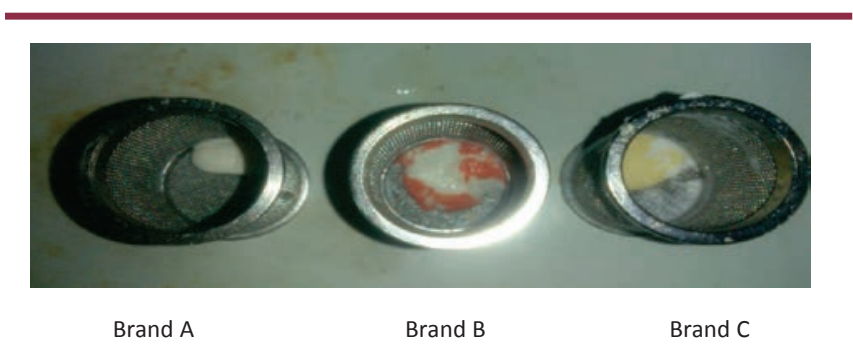

Figure 1. Partially disintegrated tablets after $1 \mathrm{~h}$.
There is a significant difference between the release profiles in the presence of milk and curd. First, Amul Taaza was the milk used for the study, and according to its label claim, each serving of $100 \mathrm{~mL}$ contains $3.1 \mathrm{~g}$ of protein and $150 \mathrm{mg}$ of calcium (6). The curd used for the study was Amul Masti Dahi, and according to its label claim (7), each serving of $100 \mathrm{~g}$ contains $4.1 \mathrm{~g}$ of protein and $183 \mathrm{mg}$ of calcium. The total fat content in both milk and curd was constant (i.e., $3 \mathrm{~g})$.

The interaction between quetiapine fumarate and calcium has been reported (8), so in the presence of calcium, drug bioavailability will decrease because of complexation with calcium. Quetiapine fumarate is a dibenzothiazepine, and an in vitro milk-drug interaction study (9) using radiolabeled benzodiazepines proved that the major fraction of binding occurs with the casein protein. Therefore, in the presence of food (milk/curd), drug bioavailability is drastically decreased not only because of quetiapine-calcium complexation but also because the adsorption of quetiapine on the surface of protein (casein) decreases the amount of absorbable quetiapine present (10). Studies (3) have indicated that the presence of milk delays tablet disintegration time. Thus, the release results for tablets in the presence of curd are much less than those in milk because of the comparatively higher calcium and protein (casein) content in curd. When FeSSGF is considered, the buffer itself contains $50 \%$ milk, which completely prevents tablet disintegration even after $1 \mathrm{~h}$, and the $0 \%$ release can be directly correlated.

The dissolution studies were then carried out with the addition of $50 \mathrm{~g}$ of egg white (equivalent to two eggs). Drug release profiles decreased less than those with milk. The dissolution release was $<30 \%$ for all three brands; there was not much variation among the individual tablets for each brand. The AUC values for Brands A, B, and C showed $58.7 \%, 78.5 \%$, and $68.7 \%$ available free drug, respectively. The release at the $Q$ point was compared using oneway ANOVA, and there was a significant difference $(p=$ $0.000)$. Furthermore, dissolution studies carried out with the addition of $50 \mathrm{~g}$ boiled egg white (equivalent to two eggs) showed drug release profiles almost similar to those with raw egg white. The dissolution release was $<25 \%$ for all three brands. In addition, there was not much variation among individual tablets for each brand. The comparative $A U C$ values for Brands $A, B$, and $C$ showed $66 \%, 64.5 \%$, and $68.9 \%$ available free drug, respectively. The release at the $Q$ point using one-way ANOVA showed 


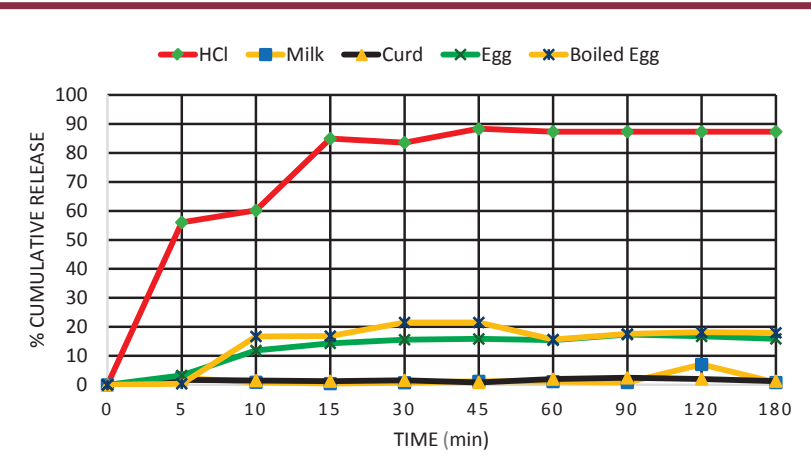

Figure 2. Comparative dissolution profiles of Brand A in various media.

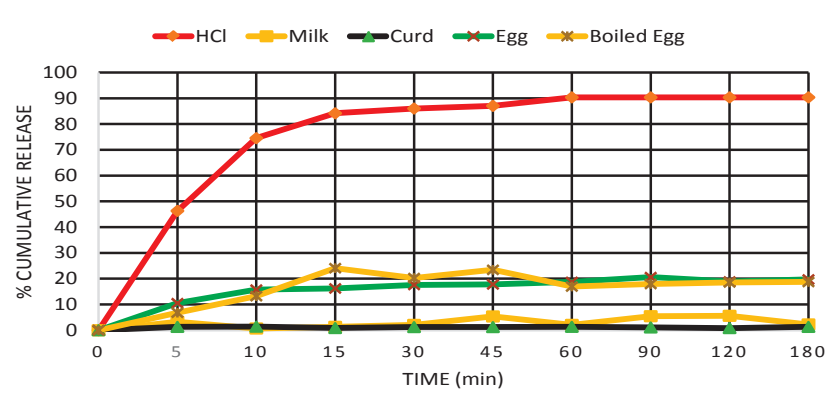

Figure 4. Comparative dissolution profiles of Brand C in various media.

a significant difference $(p=0.005)$. The comparative graphs for the release profiles in various media for Brands $A, B$, and $C$ are shown in Figures 2,3 , and 4, respectively. The comparative $A U C$ values for various media are shown in Figure 5.

Quetiapine fumarate is reported to be $83 \%$ bound to plasma proteins (albumin) (11). Similar results were obtained using egg albumin in the dissolution medium. When an egg is boiled, proteins in the egg white undergo denaturation, which results in breaking of weak bonds between the proteins such that the proteins become uncoiled. The uncoiled proteins aggregate and form new chemical bonds. The results for boiled and raw egg white were similar; hence, the boiling of an egg does not affect the drug-interaction site. The protein (albumin) may cause quetiapine fumarate to adsorb on its surface. These results show that the interaction of quetiapine with food is very structure specific.

The dissolution studies were then carried out with the addition of $200 \mathrm{~mL}$ Thums Up and Sprite (equivalent to one bottle). The drug release profiles show a slower release when compared with only $0.1 \mathrm{~N} \mathrm{HCl}$. The dissolution release for all three brands was $<85 \%$ in Thums Up and Sprite and $<80 \%$ in ENO; there was not much variation

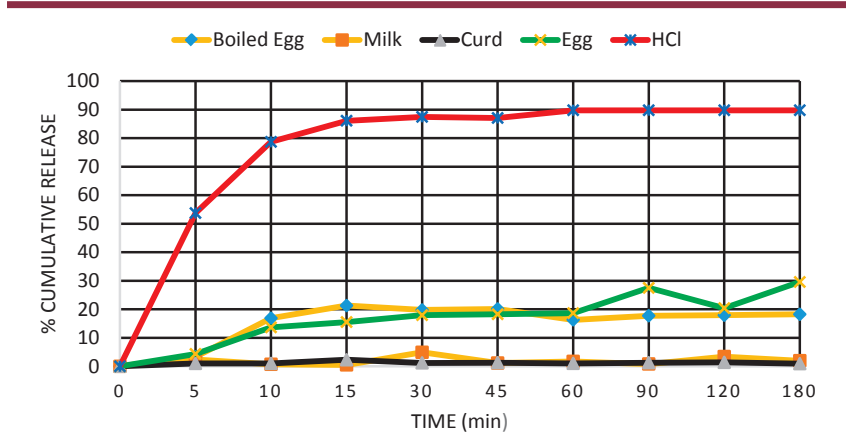

Figure 3. Comparative dissolution profiles of Brand B in various media

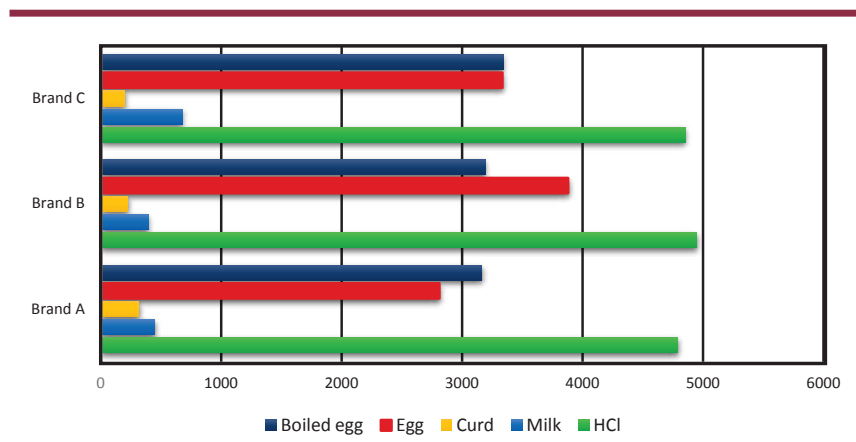

Figure 5. Comparison of AUC values for different brands in various media.

among individual tablets for each brand. The AUC values of Brands A, B, and C showed $87.8 \%, 92.4 \%$, and $83.6 \%$ available free drug for Thums Up; $84.6 \%, 91.8 \%$, and $89.4 \%$ for Sprite, and $87.3 \%, 78.9 \%$, and $64.4 \%$ for ENO, respectively. The release values at the $Q$ point were compared using one-way ANOVA, and there were no significant differences $(p=0.000)$ for Thums Up, Sprite, or ENO.

The presence of carbonated drinks causes variation in the disintegration behavior of tablets. In the presence of beverages and antacids, all three brands showed a delay in disintegration. Only Brand $A$ passed the $Q$ point in the presence of both beverages and antacids. Brands $B$ and $C$ failed to pass the $Q$ point in the presence of antacids (ENO).

\section{CONCLUSION}

The drug release profiles were evaluated in the absence and presence of protein-rich food. Drug-binding is very specific and depends upon the type and amount of protein present. The in vitro studies show that quetiapine fumarate tablets should be avoided with milk, curd, and egg (raw or boiled). The presence of protein-rich food greatly decreases the release of drug, and beverages and antacids also affect the dose that needs to be delivered to the patient. In vivo studies should be conducted to 
determine the IVIVC for similar conditions. If the IVIVC results are similar, then instructions should be included in the package insert to avoid protein-rich food and beverages (milk, curds, egg) as well as antacids so that the patient receives maximum drug availability.

\section{ACKNOWLEDGMENT}

The authors wish to thank Dr. P. G. Shrotriya, Director, Pharma Research, for his guidance.

\section{REFERENCES}

1. Indian Pharmacopoeia, Vol. 1; Indian Pharmacopoeia Commission, Ministry of Health and Family Welfare, Government of India: New Dehli, 2014.

2. Quetiapine. WebMD Web site. http://www.webmd. com/drugs/2/drug-4689-8274/quetiapine-fumarateoral/quetiapine-oral/details (accessed Jan 11, 2016).

3. Chuong, M. C.; Taglieri, C. A.; Crosby, S. J.; Ferullo, J. W.; $\mathrm{Ng}$, P. Effect of Beverages on the In Vitro Disintegration of Immediate-Release Pain Medications. Dissolution Technol. 2010, 17 (1), 31-37. DOI: 10.14227/ DT170110P31.

4. Lee, S. L.; Raw, A. S.; Yu, L. Dissolution Testing. In Biopharmaceutics Applications in Drug Development; Rajesh, K., Yu, L., Eds.; Springer: New York, 2008; pp 47-74.

5. Yu, L. X. Use and Limitations of In Vitro Dissolution Testing: Topic Introduction and Overview. Advisory Committee for Pharmaceutical Science and Clinical Pharmacology, August 8, 2012; U.S. Food and Drug
Administration Amul Taaza product details. Amul Web site. http://www.amul.com/products/amultaaza-info.php (accessed Jan 11, 2016).

6. Amul Taaza product details. Amul Web site. http:// www.amul.com/products/amul-taaza-info.php (accessed Jan 11, 2016).

7. Amul Masti Dahi product details. Amul Web site. http://www.amul.com/products/amul-mastidahiinfo.php (accessed Jan 11, 2016).

8. Review: taking Seroquel $\mathrm{Xr}$ and Calcium together. eHealthMe Web site. http://www.ehealthme.com/ drug-interactions/seroquel-xr-and-calcium (accessed Jan 11, 2016).

9. Stebler, T.; Guentert, T. W. Binding of Drugs in Milk: The Role of Casein in Milk Protein Binding. Pharm. Res. 1990, 7 (6), 633-637. DOI: 10.1023/A:1015826413335.

10. Pápai, K.; Budai, M.; Ludányi, K.; Antal, I.; Klebovich, I. In vitro food-drug interaction study: Which milk component has a decreasing effect on the bioavailability of ciprofloxacin? J. Pharm. Biomed. Anal. 2010, 52 (1), 37-42. DOI: 10.1016/j. jpba.2009.12.003.

11. Quetiapine $25 \mathrm{mg}$ film-coated tablets. electronic Medicines Compendium (eMC) Web site. http:// www.medicines.org.uk/emc/medicine/26575/SPC/ Quetiapine+25+mg+film-coated+tablets/ (accessed Jan 22, 2016). 